\title{
The use of an in vitro microneutralization assay to evaluate the potential of recombinant VP5 protein as an antigen for vaccinating against Grass carp reovirus
}

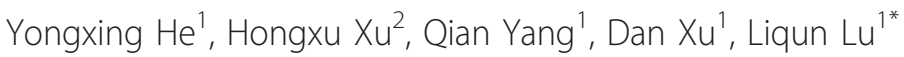

\begin{abstract}
Background: Grass carp reovirus (GCRV) is the causative pathogen of grass carp hemorrhagic disease, one of the major diseases damaging grass carp Ctenopharyngon idellus breeding industry in China. Prevention and control of the disease is impeded largely due to the lack of research in economic subunit vaccine development. This study aimed to evaluate the potential of viral outer shell protein VP5 as subunit vaccine.

Methods: The vp5 gene was isolated from the viral genome through RT-PCR and genetically engineered to express the recombinant VP5 protein in E coli. The viral origin of the recombinant protein was confirmed by Western blot analysis with a monoclonal antibody against viral VP5 protein. Polyclonal antibody against the recombinant VP5 protein was prepared from mice. A microneutralization assay was developed to test its neutralizing ability against GCRV infection in cell culture.
\end{abstract}

Results: The GST-VP5 fusion protein (VVP5) was produced from E. Coli with expected molecular weight of $90 \mathrm{kDa}$. The protein was purified and employed to prepare anti-VP5 polyclonal antibody from mice. The anti-VP5 antibody was found to neutralize GCRV through in vitro microneutralization assay and viral progeny quantification analysis.

Conclusions: The present study showed that the viral VP5 protein was involved in viral infection and bacteriallyexpressed VP5 could be suitable for developing subunit vaccine for the control of GCRV infection.

\section{Background}

Reoviruses are distributed widely in aquatic environments and have been isolated from a wide range of aquatic organisms. Grass carp reovirus (GCRV) is currently one of the most serious pathogens threatening the grass carp Ctenopharyngon idellus production with high mortality in China [1]. The virions consist of a double-layered protein capsid containing 11 dsRNA genomic fragments [2]. GCRV was assigned to the genus Aquareovirus of the family Reoviridae by the international committee on Taxonomy of Viruses (ICTV) in 1991 [3]. It differed from orthoreovirus in a number of characteristics such as absence of an

\footnotetext{
* Correspondence: Iqlv@shou.edu.cn

'Key Laboratory of Aquatic Genetic Resources and Utilization/Ministry of

Agriculture, Shanghai Ocean University, 201306 Shanghai, China

Full list of author information is available at the end of the article
}

antigenic relationship and unequal numbers of genome segments [4]. To improve the production of grass carp and reduce the economic losses, effective vaccine against GCRV is urgently desired for the fish cultivation industry. However, functional characterization of the encoding proteins of GCRV has been limited due to the lack of research interest of GCRV in regions outside of China. Besides this, the majority of human orthoreovirus infections involve the gastrointestinal and upper respiratory, which are generally asymptomatic [5]. The majority of adults have neutralizing antibodies and no preventative vaccination is needed for the viral infection. Thus, although extensive studies have been conducted on the replication of mammalian reovirus in host cells, little effort has been made to test the vaccine potential of its structural proteins. Fully attenuated apathogenic avian reovirus vaccine did have been developed by serial

\section{C) Biomed Central}


passage of virus in chicken eggs and chicken embryo fibroblast cultures [6,7]. Outer capsid Sigma C protein had been implicated for the use of potential subunit vaccine against avian reovirus infection [8]. Until now, the only commercial carp vaccine in Asia is an inactivated grass carp reovirus vaccine [9]. Traditional methods, such as attenuation of wild-type viruses to generate live vaccines and formalin-inactivation to produce killed vaccine, are still being employed to develop effective preventive strategy against GCRV in China [10]; the unpopular application of these vaccines at present indicates that further improvement of vaccines in terms of safety, efficacy, manufacturing cost, and field manipulation is essential for the disease control. New advances in molecular biology and biotechnology of virus could help us understand which viral factors are important for induction of strong immunity and lead to new strategies of vaccine design [11].

Identification and production of protective antigens is probably the most feasible strategy towards low-cost vaccines for low-price grass carp. The core of GCRV is composed of five proteins: VP1, VP2, VP3, VP4 and VP6 [12]. A total of 120 VP3 molecules form the spherical inner capsid shell of the GCRV inner core. The outer capsid of GCRV was composed of 200 trimers of VP5-VP7 heterodimers, which were analogous to the $\mu 1_{3} \sigma 3_{3}$ complexes of well-characterized mammalian reovirus but with significant differences in protein structure and low homology. VP7 only shares a very low sequence identity of $12 \%$ with its counterpart $\sigma 3$ of Mammalian reovirus, while the identity between VP5 and $\mu 1$ is $24 \%$ [13]. The outer capsid proteins of both mammalian reovirus and GCRV were involved in host recognition and attachment during viral replication although not required for efficient viral replication inside the host cells $[14,15]$. The function of VP7 is unclear at present, while the overall similar structure between VP5 and $\mu 1$ suggests a similar functional role of cell membrane penetration during viral entry into host cells for both proteins [13]. Polyclonal antibodies against $\mu 1$ and $\sigma 3$ were reported able to neutralize Mammalian reovirus in vitro [14]; thus, among all the five viral structural proteins, VP5 was selected to test its subunit vaccine potential against GCRV in this report.

The aim of present study was to evaluate the potential of VP5 protein as subunit vaccine against GCRV infection through employing in vitro microneutralization assays, which has been used successfully for the measurement of neutralizing antibodies to various viruses in vaccine and epidemiologic studies [16]. Information derived from such assays formed the basis for passive immunization strategies against virus infection $[17,18]$. The investigation also present information on the basic role of VP5 protein in the initial steps of GCRV infection.

\section{Results}

Expression, purification and immunoblotting analysis of recombinant VP5 protein (rVP5)

To produce rVP5 in E. coli, the vp5 gene fragment of 1947 bp was isolated through RT-PCR from the purified viral dsRNA genome (Figure 1a). The PCR product was then inserted to the bacterial expression vector pGEX3T-4 for the expression of a GST-VP5 fusion protein, the correct clone bearing the indeed vp5 gene was confirmed by gene sequencing. Expected GST-VP5 fusion protein of about $90 \mathrm{KDa}$ could be induced by IPTG in cultured bacterial (Figure 1b). The expression level of rVP5 was about $10 \%$ of the total bacterial protein. The IPTG-induced protein of $50 \mathrm{ng} / \mu \mathrm{l}$ was isolated from the gel for the use of purified antigen to develop polyclonal antibodies against VP5 (Figure 1b). The viral origin of the induced and purified rVP5 was confirmed by immunoblotting assay with a specific monoclonal antibody against viral VP5 protein (Figure 1c).

\section{Specificity of polyclonal antibody against GCRV virions}

To prepare polyclonal antibodies against GCRV VP5 protein, the purified rVP5 was injected into the mice as described in the Materials and Methods. However, it was unclear whether the rVP5 sustained the same antigenicity as viral VP5 protein. Thus, the raised polyclonal antibodies were pooled together, named as anti-VP5 here, for the determination of its specificity toward GCRV viral particles by immunoblotting assay. Figure 2 showed that the pooled polyclonal antibody recognized the viral VP5 protein as revealed by the positive signal at the expected size of about $67 \mathrm{KDa}$. In contrast, the control serum contained no antibody to recognize viral VP5 protein in the immunoblotting assay (Figure not shown).

\section{Microneutralization assay and virus titration}

The above results indicated that anti-VP5 polyclonal antibody was able to recognize the viral VP5 protein, but it remained to know whether it contained antibodies that could block the virus infection for evaluation of the potential of rVP5 as subunit vaccine. For this purpose, a microneutralization assay was employed to check the neutralizing ability of the anti-VP5 polyclonal antibody. For more accuracy, the serum dilution was tested in four replicate wells per dilution (Figure 3a). Based on the visual assay result, the neutralization titer of the tested antibody was 1:40.

To confirm the neutralization result, the supernatant of infected cells in the 96 well plate was collected for viral titration assay. Low viral progeny of $10^{2}-10^{3}$ TCID50/ml was observed for the wells infected with GCRV mixed with anti-VP5 sera of 1:20 or 1:40 dilution; while the viral production reached $10^{7} \mathrm{TCID} 50 / \mathrm{ml}$ for the cells 


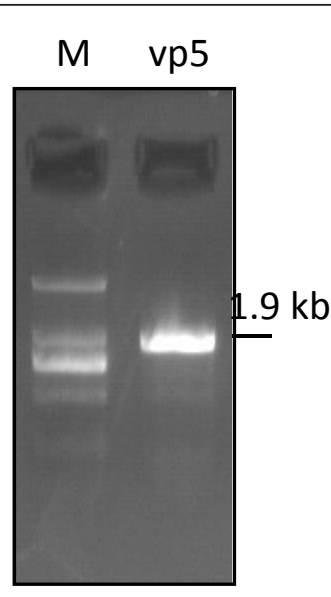

a

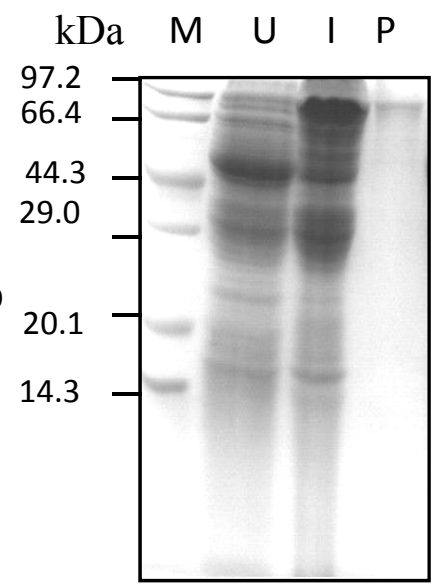

b

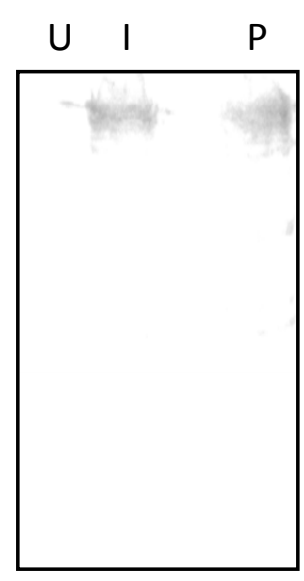

C

Figure 1 Cloning, expression, purification and immunoblotting assay of rVP. a, the 1\% agarose gel electrophoresis of vp5 gene fragment amplified from GCRV genome by RT-PCR. b, 10\% SDS-PAGE analysis of induced and purified rVP5. c, Immunoblotting assay of rVP5 with monoclonal antibody against VP5 protein. Second antibody was the HRP-conjugated goat-anti-mouse (Sigma). M, Protein marker; U, total cell extract of uninduced bacterial; I, total cell extract of IPTG-induced bacterial; P, purified rVP5.

infected with GCRV when mixed with anti-VP5 sera of 1:80 to $1: 2560$ dilution (Figure $3 \mathrm{~b}$ ).

\section{Discussion}

Grass carp Ctenopharyngon idellus culture is an important economic industry for China, which has suffered from GCRV-induced hemorrhagic disease for some years [19]. Although whole virus-inactivated vaccine had been applied in some fish farms for the prevention of the disease, more efficient and economic vaccine approaches were urgently desired for the national-wide control of the viral disease. Subunit vaccine presents an ideal promise for this purpose. The present study aimed

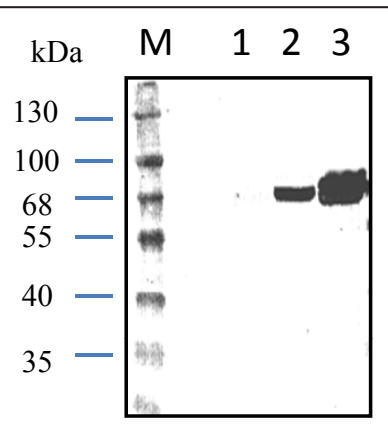

Figure 2 Immunoblotting assay of GCRV virions with polyclonal antibody anti-VP5. Mock-infected CIK cell lysate from $10^{5}$ cells (lane 1), purified GCRV viral particles of $1 \mu \mathrm{g}$ (lane 2) and GCRV-infected CIK cell lysate from $10^{5}$ cells (lane 3 ) were subjected for $10 \%$ SDS-PAGE analysis and transferred to PVDF membrane. The first antibody used here was the pooled antisera collected from the immunized mice. Second antibody was the HRP-conjugated goatanti-mouse (Sigma). to identify the subunit vaccine potential of outer shell protein VP5. As the outer shell protein, VP5 might have key effects on the viral infection, such as recognition and attachment to receptors in the host cell surface, as well as penetration into the host cell membrane during the virus assembly [20]. The fact that anti-VP5 polyclonal antibodies efficiently blocked viral infection here supported that VP5 was involved in viral infection, and posed as a good candidate for the development of subunit vaccine.

The availability of a stable grass carp cell line CIK cells and the strong CPE induced by GCRV infection paved the way for the establishment of an in vitro neutralization assay. For the visual microneutralization assay, an optimal working dilution of $50 \mathrm{TCID}_{50}$ virus in $50 \mu \mathrm{l}$ volume per well was determined in a preliminary logarithmic serial dilution tests. It ensured observable CPE within $48 \mathrm{~h}$ postinfection under light microscope in contrast to the uninfected cells. The stained signal of cells in the 96 well plate irreversibly correlated well with the virus-induced CPE for the low dilution of sera (1:20$1: 80$ ), which validated the method for neutralization assay. However, for the sera of high dilution (1:160 $1: 2560)$, the difference of stained signal was not significant, which was reasonable due to the fact that un-neutralized virus replicated quickly in CIK cells. We didn't optimize the production procedure of anti-VP5 antibody from mice in this study. The low neutralizing titer of 1:40 might be largely due to the low titer of anti-VP5 polyclonal antibody raised from mice. To further validate the visual neutralization assay, virus end-point titers were calculated using $\mathrm{TCID}_{50}$ assay. 


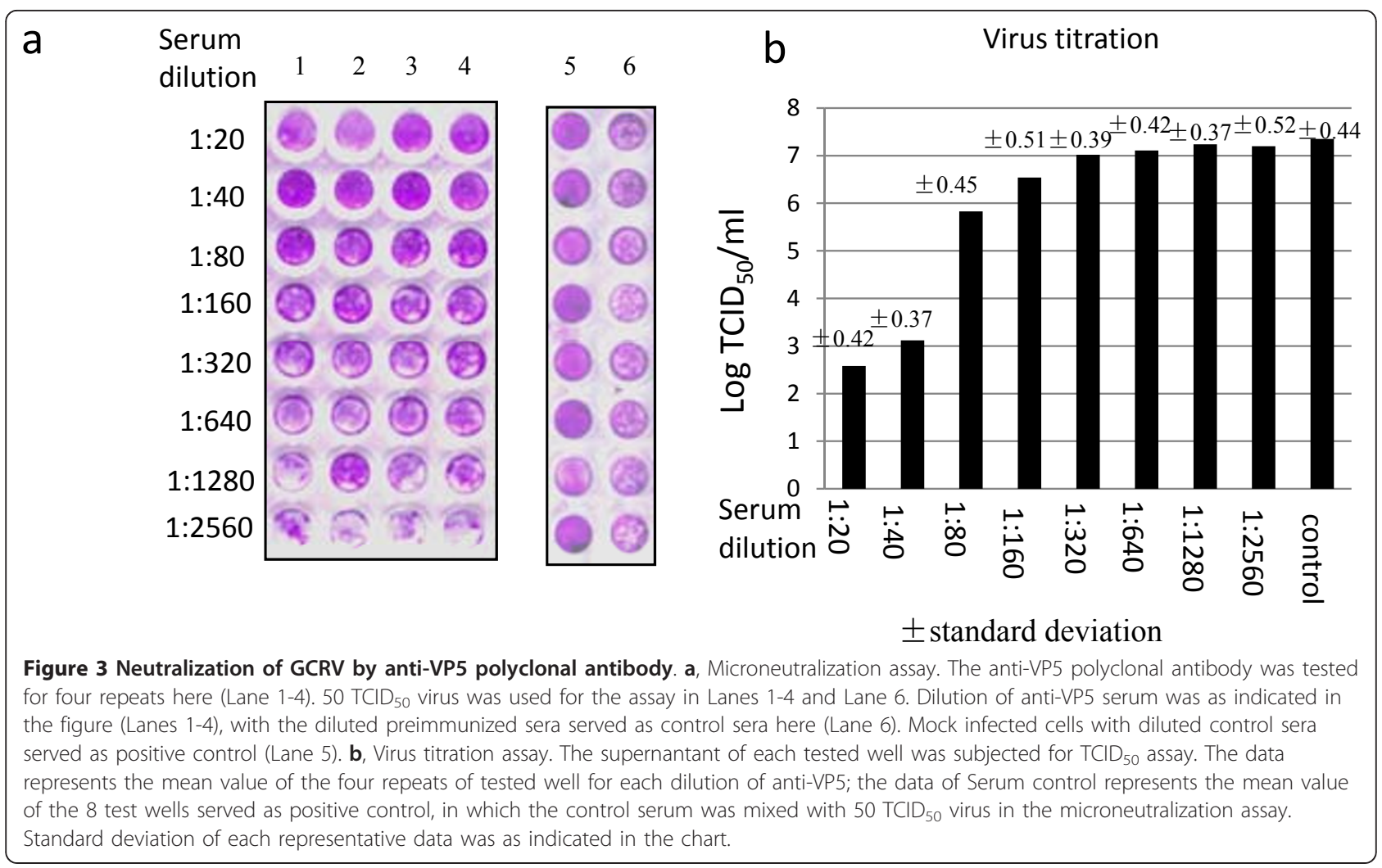

The TCID $_{50}$ value of the infected supernatant of each tested well correlated very well with the amount of CPE as showed by the reduction of stained signal of the cell monolayer. It was worthy to note that, in the microneutralization assay, the continued presence of antibody through the course of the assay could neutralize virus released from infected cells.

As far is known, this is the first report on designing a microneutralization assay to test neutralization antibodies against GCRV. The assay could test antibody samples with high throughput since the cell culture, viral infection and staining were all performed in a 96-well format. For further in vivo animal experiments to test the protective effect of rVP5 protein, the microneutralization assay on a large scale could still be valuable in addressing the protection mechanism on how and when the neutralization antibody was produced in individual immunized animal. This assay could also be employed for mapping neutralizing epitope of VP5 protein for immunological characterization purpose.

In summary, the gene of GCRV outer shell protein VP5 was cloned and efficiently expressed in E coli. to get recombinant protein antigen $\mathrm{rVP} 5$, an in vitro microneutralization assay was developed to show that polyclonal antibody against rVP5 was able to block viral infection. The results support the idea that a subunit vaccine based on the rVP5 was possible for the control of GCRV infection in Grass carp Ctenopharyngon idellus.

\section{Methods}

Virus culture

Grass carp Ctenopharyngon idellus kidney cells (CIK) were maintained in DMEM medium with $10 \%$ fetal bovine serum [21](Zuo et al., 1984). Grass carp reovirus 873 strain (prototype strain) from CCTCC (China center for type culture collection) was used in the study. To propagate the virus, monolayers of CIK were infected with GCRV and incubated for 2-3 days at $28^{\circ} \mathrm{C}$. Infected supernatants or cells were harvested when greater than 90\% CPE (Cytopathic effect) was observed.

\section{Viral RNA extraction and RT-PCR reaction}

Culture supernatant $(250 \mathrm{ml})$ containing GCRV virions were collected and centrifuged at $60,000 \times \mathrm{g}$ for $1 \mathrm{~h}$ (SW41 rotor, Beckman). Genomic dsRNA was then extracted from the purified GCRV using a Trizol method (Invitrogen). The dsRNA was separated from contaminating ssRNA by precipitating in $2 \mathrm{M}$ lithium chloride $(\mathrm{LiCl})$. The dsRNA was resuspended in DEPC-treated water and stored at $-80^{\circ} \mathrm{C}$ until use. The reverse transcription was performed with the reverse transcription kit (Takara) using the extracted viral genome template and random primer according to the manufacture's protocol. 
The synthesis of the cDNA was carried out in a reverse transcriptase reaction mixture at $42^{\circ} \mathrm{C}$ for $60 \mathrm{~min}$ and stopped by the addition of $0.5 \mathrm{M}$ EDTA, pH8.0. The cDNA was used directly for PCR amplification of vp5 gene using Master PCR system (Takara). Vp5 gene was amplified through PCR using primer pairs of TCCCCCGGGGGATGGGGAACGTTCAAACCTCCGT and ATAAGAATGCGGCCGCTTATCACTTGCCGGG CCACAA from the viral genome cDNA mix. Amplification was carried out after denaturation at $94^{\circ} \mathrm{C}$ for $2 \mathrm{~min}$, followed by 30 cycles of denaturation for $15 \mathrm{~s}$ at $95^{\circ} \mathrm{C}$, annealing for $30 \mathrm{~s}$ at $62^{\circ} \mathrm{C}$, extension for $2 \mathrm{~min}$ at $68^{\circ} \mathrm{C}$ and a final extension step for $10 \mathrm{~min}$ at $72^{\circ} \mathrm{C}$. After sequence confirmation, the vp5 gene fragment was digested by Sma I and Not I before inserting into the corresponding restriction sites of pGEX4T-3 (GE Healthcare) to get recombinant plasmid pGEX-vp5.

\section{Expression of recombinant VP5 protein and Western blot assay}

To express VP5 as glutathione (GST) fusion protein by pGEX-vp5 in DH5 $\alpha, 100 \mathrm{ml}$ of Luria-Bertani medium containing $100 \mu \mathrm{g}$ ampicillin/ml was inoculated with $1 /$ 100 of an overnight culture containing either of the recombinant plasmids and grown to an optical density of 0.6 at $600 \mathrm{~nm}$. Protein expression was induced with 1 mM IPTG (isopropyl- $\beta$-D-thiogalactopyranoside) for 3 h. The bacteria were collected at $6000 \times \mathrm{g}$ for $10 \mathrm{~min}$, and the pellet was resuspended in $5 \mathrm{ml}$ of PBS. Protein expression level was judged by SDS-PAGE, through which the ChemiDoc ${ }^{\mathrm{TM}} \mathrm{XRS}+$ system (Bio-Rad) was employed for the automated quantitative analysis of stained protein samples on PAGE gel. The specificity and viral origin of expressed protein was further analyzed by Western Blot assay using specific monoclonal antiserum again VP5, which was commercially developed using standard methods by injection of specific peptide sequence (291aa-305aa: PRSYRPAFIKPEDAK) of VP5 into mouse at the antibody production facility in AbMart, China. For Western blots, proteins were resolved by SDS-PAGE and transferred to PVDF (Biorad) using standard methods. After blocking in 4\% milk and binding of primary antibodies, membranes were washed extensively and incubated with anti-mouse IgG conjugated to alkaline phosphatase. Signal was development by using BCIP and NBT substrates (Sigma).

\section{Purification of VP5 protein and preparation of polyclonal antibody}

The insoluble debris containing the GST-VP5 fusion protein was collected by centrifugation at 5,000 g and washed three times using PBS. Then the total insoluble protein was subjected to SDS-polyacrylamide gel electrophoresis (SDS-PAGE) analysis, then cut from the gel and used for protein purification with PAGE gel protein recovery kit (Sangon Biotech). To generate specific polyclonal antisera towards VP5, groups of 4 adult (6 weeks old) female Balb/c mice were intraperitoneally immunized in a $50 \%$ emulsion of Freud's adjuvant with $50 \mu \mathrm{g}$ of purified recombinant VP5 protein, and boosted twice at 3 weeks interval with the same doses. Immune sera were collected 7 days after the last immunization. Pre-immune serum was collected before immunization and used as a control in neutralization experiments. Immunoglobulin (IgG) antibodies were purified by protein A-sepharose (Promega) and stored at $-20^{\circ} \mathrm{C}$. Both pre-immune sera and immunized sera were pooled together in the study, which were named as serum control and anti-VP5 serum respectively.

$\mathrm{TCID}_{50}(50 \%$ tissue culture infective dose) assay

The GCRV titer was determined as $\mathrm{TCID}_{50}$ on CIK cells based on a typical cytopathic effect produced by viral infection [22]. CIK cells were maintained as stock cultures in DMEM and re-plated 2 days before infection with GCRV in 96 well plates for TCID $_{50}$ assay. The virus stock was serially diluted with DMEM and then used for infection of CIK. Cell cultures were infected for $1 \mathrm{~h}$, and fed with $200 \mu \mathrm{l}$ fresh medium. $48 \mathrm{~h}$ post infection, 96 well plates were observed under light miscope for typical CPE. The $\mathrm{TCID}_{50}$ value was calculated using Reed Muench method.

\section{Microneutralization assay}

The in vitro microneutralization assay was performed according to a modified protocol [23]. Cells infected with $50 \mathrm{TCID}_{50}$ virus and cells with mock infection served as positive and negative controls in the assay respectively. The assay medium for serum and virus dilution was the complete growth medium for CIK cells. The sera were inactivated at $56^{\circ} \mathrm{C}$ for $30 \mathrm{~min}$ and serially diluted twofold in a microtiter plate with $50 \mu \mathrm{l}$ per well. Six serial 2-fold dilutions (1:20 - 1:2560) of serum in a volume of $50 \mu \mathrm{l}$ were loaded into the plate wells, followed by the addition of $50 \mathrm{TCID}_{50}$ virus. The plates were incubated at $28^{\circ} \mathrm{C}$ for $1 \mathrm{~h}$. During the incubation period, CIK cells were trypsinized and resuspended at 5 $\times 10^{4}$ cells per ml. Cell suspension $(100 \mu \mathrm{l}$ per well) was added to the plates, the solutions were mixed, and the plates were incubated at $28^{\circ} \mathrm{C}$ for $48 \mathrm{~h}$. Cells in the 96 well plate were then stained with the dye crystal violet (5\% in PBS), which stains only living cells here. If a serum sample contains antibodies that block viral infection, most of the cells will survive and present violet color; if the virus can't be blocked by the serum, cells will be infected, round up and detach from the cell culture plate, thus no violet staining is visible for infected 
cells. The neutralization titer is expressed as the reciprocal of the highest dilution at which virus infection is blocked.

\section{Acknowledgements}

The project was funded by the Shanghai committee of Science and Technology (Grant No. 08390510200 and 10PJ1404800). Lu L. was also supported by the earmarked fund for China Agriculture Research System and the Program for Professor of Special Appointment (Eastern Scholar) at Shanghai Institutions of Higher Learning.

\section{Author details}

'Key Laboratory of Aquatic Genetic Resources and Utilization/Ministry of Agriculture, Shanghai Ocean University, 201306 Shanghai, China. ${ }^{2}$ Department of Medical Diagnostic, Zhongshan School of Medicine, Sun Yat-sen University, Guangzhou 510080, China.

\section{Authors' contributions}

$\mathrm{LL}$ designed the experiments and drafted the manuscript. YH, HX and QY performed most of the experiments. DX assisted in molecular cloning and protein analysis. All authors have read and approved the final manuscript.

\section{Competing interests}

The authors declare that they have no competing interests.

Received: 20 January 2011 Accepted: 22 March 2011

Published: 22 March 2011

\section{References}

1. Qiu T, Lu R, Zhang J, Zhu Z: Complete nucleotide sequence of the $\mathrm{S} 10$ genome segment of grass carp reovirus (GCRV). Dis Aquat Org 2001, 44:69-74.

2. Zhang C, Wang Q, Shi C, Zeng W, Liu Y, Wu S: Molecular analysis of grass carp reovirus HZO8 genome segments 1-3 and 5-6. Virus Genes 2010, 41(1):102-104.

3. Francki RIB, Fauquet CM, Knudson DL, Brown F: Classification and Nomenclature of Viruses. Fifth Report of the International Committee on Taxonomy of Viruses Wien: Springer-Verlag; 1991.

4. Attoui H, Fang Q, Jaafar FM, Cantaloube J, Biagini P, Philippe dM, Lamballerie $X$ : Common evolutionary origin of aquareoviruses and orthoreoviruses revealed by genome characterization of Golden shiner reovirus, Grass carp reovirus, Striped bass reovirus and golden ide reovirus (genus Aquareovirus, family Reoviridae). J Gen Virol 2002, 83:1941-1951.

5. Tyler KL: Pathogenesis of reovirus infections of the central nervous system. Seminars in Neuroscience 1991, 3(2):117-124.

6. Van der Heide L, Kalbac M, Brustolon M: Development of an attenuated apathogenic reovirus vaccine against viral arthritis/Tenosynovitis. Avian Dis 1983, 27(3):698-706.

7. Guo ZY, Giambrone JJ, Wu H, Dormitorio T: Safety and Efficacy of an Experimental Reovirus Vaccine for In Ovo Administration. Avian Dis 2003, 47(4):1423-1428

8. Vasserman $Y$, Eliahoo D, Hemsani E, Kass N, Ayali G, Pokamunski S, Pitcovski J: The Influence of Reovirus Sigma C Protein Diversity on Vaccination Efficiency. Avian Dis 2004, 48(2):271-278.

9. Sommerset I, Krossøy B, Biering E, Frost P: Vaccines for fish in aquaculture. Expert rev vaccines 2005, 4(1):89-101.

10. Zeng L, Yang X, He L, Ai X, He J, Zuo W: Batch processing technology of cell cultured killed vaccine against the hemorrhage of Grass carp. $J$ Fishery Sci China 1998, 5(2):62-67.

11. Ellis AE: Immunity to bacteria in fish. Fish Shell Immunol 1999, 9(4):291-308.

12. Cheng L, Zhu J, Hui WH, Zhang X, Honig B, Fang Q, Zhou ZH: Backbone Model of an Aquareovirus Virion by Cryo-Electron Microscopy and Bioinformatics. J Mol Biol 2009, 397(2):852-863.

13. Cheng L, Fang Q, Shah S, Atanasov IC, Zhou ZH: Subnanometer-Resolution Structures of the Grass Carp Reovirus Core and Virion. J Mol Biol 2008, 382(1):213-222.
14. Chandran K, Walker SB, Chen Y, Contreras CM, Schiff LA, Baker TS, Nibert ML: In vitro recoating of reovirus cores with baculovirusexpressed outer-capsid proteins $\mu 1$ and 3. J Virol 1999, 73:3941-3950.

15. Fan C, Shao L, Fang Q: Characterization of the nonstructural protein NS80 of grass carp reovirus. Arch Virol 2010, 155(11):1755-1763.

16. Andreoni M, Faircloth $M$, Vugler L, Britt W: A rapid microneutralization assay for the measurement of neutralizing antibody reactive with human cytomegalovirus. J Virol Meth 1989, 23(2):157-167.

17. Pastrana DV, Vass WC, Lowy DR, Schiller JT: NHPV16 VLP Vaccine Induces Human Antibodies That Neutralize Divergent Variants of HPV16. Virology 2000, 279(1):361-369.

18. Schofield DJ, Glamann J, Emerson SU, Purcel RH: Identification by Phage Display and Characterization of Two Neutralizing Chimpanzee Monoclonal Antibodies to the Hepatitis E Virus Capsid Protein. J Virol 2000, 74(12):5548-5555.

19. Rangel AA, Rockemann DD, Hetrick FM, Samal SK: Identification of grass carp haemorrhage virus as a new genogroup of aquareovirus. J Gen Virol 1999, 80:2399-2402.

20. Fang Q, Seng EK, Ding QQ, Zhang LL: Characterization of infectious particles of grass carp reovirus by treatment with proteases. Arch Virol 2008, 153(4):675-682

21. Zuo WG, Qian HX, Xue YF: Establishment of grass carp kidney cell line (CIK), Fresh-water Fisheries. 1984, , 2: 38-39, (in Chinese)

22. LaBarre DD, Lowy RJ: Improvements in methods for calculating virus titer estimates from TCID50 and plaque assays. J Virol Methods 2001, 96(2):107-126

23. Crawford-Miksza LK, Schnurr DP: Quantitative colorimetric microneutralization assay for characterization of Adenovirus. J Clin Microbiol 1994, 32(9):2331-2334.

doi:10.1186/1743-422X-8-132

Cite this article as: He et al:: The use of an in vitro microneutralization assay to evaluate the potential of recombinant VP5 protein as an antigen for vaccinating against Grass carp reovirus. Virology Journal 2011 8:132.

\section{Submit your next manuscript to BioMed Central and take full advantage of:}

- Convenient online submission

- Thorough peer review

- No space constraints or color figure charges

- Immediate publication on acceptance

- Inclusion in PubMed, CAS, Scopus and Google Scholar

- Research which is freely available for redistribution 